\title{
Wavelet and Co-occurrence Matrix based Rotation Invariant Features for Texture Image Retrieval using Fuzzy Logic
}

\author{
Shailendrakumar M. \\ Mukane \\ Department of Electronics \\ and Telecommunication \\ Engg. \\ SVERI's College of \\ Engineering, Pandharpur, \\ Maharashtra State, India.
}

\author{
Dattatraya S. Bormane \\ Department of Electronics \\ and Telecommunication \\ Engg. \\ JSPM's Rajarshi Shahu \\ College of Engineering, \\ Pune, Maharashtra State, \\ India.
}

\author{
Sachin R. Gengaje \\ Department of Electronics \\ Engg. \\ Walchand Institute of \\ Technology, Solapur, \\ Maharashtra State, India
}

\begin{abstract}
In this paper, research carried out to test the wavelet and cooccurrence matrix based features for rotation invariant texture image retrieval using fuzzy logic classifier. Energy and Standard Deviation features of DWT coefficients up to fifth level of decomposition and eight features are extracted from cooccurrence matrix of whole image and each sub-band of first level DWT decomposition. The texture image is rotated in six different angle. Each rotated texture image sampled to the $128 \times 128$, and $256 \times 256$ size. The suitability of features are tested using a fuzzy logic classifier. The performance is measured in terms of Success Rate. Success rate is calculated for each rotated texture samples and each of the feature sets. The minimum number of features required for maximum average success rate is obtained. The research shows that for samples of $256 \times 256$ size, excellent success rate is achieved for all rotation angle with Wavelet Statistical Features (WSF) as well as Wavelet Cooccurrence Features (WCF). Also energy features perform better than standard deviation features for every rotation angle considered. Also worst case analysis shows that energy features never fail to classify for any of the texture image and more consistent than other features, during the experiment. 8 cooccurrence feature set performs better than 5 co-occurrence feature set. For both the types of features performance degrades in case of $128 \times 128$ sample size.
\end{abstract}

\section{General Terms}

Content based Texture Image Retrieval, Texture Analysis, and Fuzzy logic.

\section{Keywords}

Discrete Wavelet Transform, Wavelet Statistical features, Wavelet Co-occurrence matrix features, Rotation Invariance, Fuzzy Classifier.

\section{INTRODUCTION}

Generation of digital images and its use is rapidly increasing everyday life of peoples. To access digital library information i.e. available in the form of digital images, it has to be organized properly so as to allow efficient browsing, searching, and retrieval of useful images. Therefore, Image Retrieval becomes an active research area.
The drawbacks of manual browsing, searching, and retrieval, can be reduced by Content Based Image Retrieval (CBIR) method where in images are expressed by their visual content of images. Texture, Shape, and Color are the general visual content features of image. Texture features are very important because it is an intrinsic property of virtually all surfaces such as skin, bricks, tree, fabric, grass, hair, clouds, etc. It contains information about the structural arrangement of surfaces and their relationship to the surrounding environment [1].

Weszka et al. [2] compared the classification performance of Fourier power spectrum, second order Gray Level Cooccurrence Matrix (GLCM), and first order statistics of gray level differences. It is tested for terrain samples and commented that Fourier methods performed poorly. Haralick [1] suggested GLCM texture features and used these features to analyze remotely sensed images. Wan et al. [3] presented comparative study of four texture analysis methods such as gray level Runlength method[RLM], Co-occurrence matrix method, Histogram method, and Auto-correlation method and shown that Cooccurrence method is superior. Gabor transform is a special case of Short Time Fourier Transform (STFT). Manjunath and Ma [4] had given a comprehensive performance evaluation of Gabor Wavelet based texture analysis and commented that they are quite robust. Wavelet Transform [5, 6] provides a multiresolution approach for the problem at hand. Smith and Chang [7] used mean and variance extracted from wavelet sub-band coefficients, as the texture representation.

Classification methods can be divided into categories such as parametric, non-parametric, stochastic methods, non-metric methods [8]. Classification task involves classifying images based on the feature vectors provided by the feature extraction methods. If no prior parameterized knowledge about the probability structure then classification is based on nonparametric techniques. That classification will be based on information provided by training samples alone. These techniques include fuzzy classification, neural network approach, etc. Engin Avci [9] used multilayer perceptron neural network classifier to classify selected texture images. I.Turkoglu, E. Avci [10] presented a comparison of wavelet support vector machine (W-SVM) and wavelet-adaptive network based fuzzy inference system (W-ANFIS) approaches 
for texture image classification. Both W-SVM and W-ANFIS methods are used for classification of the 22 texture images. G. Schaefer et al. [11] used fuzzy classification for thermograph based breast cancer analysis using statistical features. Wan [3] used 1-Nearest Neighbor \& k- Nearest Neighbor techniques to classify the Bark texture images and shown that 1- Nearest neighbor classifier is more appropriate than others. Mukane et al. carried out the scale invariance [12] and size invariance [13] with wavelet and co-occurrence matrix based features using fuzzy logic classifier.

In this study, it is proposed study the features for rotation invariant texture image retrieval using fuzzy logic. 25 texture images are taken from the Brodatz texture Album. Two types of feature sets are extracted in the feature extraction process viz., discrete wavelet transform based feature set, and wavelet and co-occurrence matrix based feature set. Then the average success rate and minimum features required for the maximum success rate are studied with the help of fuzzy classifier to classify texture images for samples of different angle rotated texture images.

\section{FEATURE EXTRACTION}

The texture features are extracted using DWT at different level and co-occurrence matrix of whole image and first level of DWT decomposition.

\subsection{Wavelet Statistical Features (WSF)}

The Wavelet transform provides a multi-resolution approach. It decomposes a signal with a family of basis functions obtain through translation and dilation of a mother wavelet. In this the advantage of variable window size is available. The window size can be kept wide for low frequencies and narrow for high frequencies which lead to an optimum time-frequency resolution for complete frequency range. When applied to image, DWT decomposes the image into four sub-bands followed by the subsampling.

Based on the available wavelet coefficients, Energy (1) and standard deviation (2) of all the sub-bands up to fifth level of decomposition are calculated as features by using the equation

$$
\begin{aligned}
E_{k} & =\frac{1}{N^{2}} \sum_{i=1}^{N} \sum_{j=1}^{N}\left|x_{k}(i, j)\right| \\
\sigma_{k} & =\left[\frac{1}{N^{2}} \sum_{i=1}^{N} \sum_{j=1}^{N}\left(x_{k}(i, j)-\mu_{k}(i, j)\right)^{2}\right]^{\frac{1}{2}}
\end{aligned}
$$

where $E_{k}$ is the energy \& $\sigma_{k}$ is the standard deviation for the kth sub-band of dimension NxN and coefficients are $x_{k}(i, j) \&$ mean value is $\mu_{k}(i, j)$ [14].

For each samples of different rotation angle texture image, above features are computed and stored in the data base feature vector as Wavelet Statistical Features (WSF). This feature is used at the time of classification stage.

\subsection{Wavelet Co-occurrence features (WCF)}

The Co-occurrence matrix features are obtained from whole sample image and one level DWT decomposed sub-bands coefficients of sample image. Co-occurrence matrix is derived for distance vector $d(i, j)$ i.e. offset is taken as $d(0,1)$. From the co-occurrence matrix the co-occurrence parameters namely contrast, inverse difference moment, energy, norm entropy, local homogeneity, cluster shade, cluster prominence, \& maximum probability are obtained $[9,15]$ by the equations (3) - (10) respectively.

$$
\begin{gathered}
\text { Inverse difference moment }=\begin{array}{c}
\sum_{i=1}^{N} \sum_{j=1}^{N} \frac{\operatorname{Co}(i, j)}{|i-j|^{2}} \\
\mathrm{i} \neq \mathrm{j}
\end{array} \\
\text { contrast }=\sum_{i=1}^{N} \sum_{j=1}^{N}(i-j)^{2} \operatorname{Co}(i, j) \\
\text { energy }=\sum_{i=1}^{N} \sum_{j=1}^{N} \operatorname{Co}^{2}(i, j) \\
\text { norm entropy }=\frac{\sum_{i, j=1}^{N}|\operatorname{Co}(i, j)|^{1.5}}{N} \\
\text { local homogeneity }=\sum_{i, j=1}^{N} \frac{1}{1+(i-j)^{2}} \operatorname{Co}(i, j) \\
\text { cluster shade }=\sum_{i, j=1}^{N}(i-P x+j-P y)^{3} \operatorname{Co}(i, j) \\
\sum_{i, j}^{N}(i-P x+j-P y)^{4} \operatorname{Co}(i, j)
\end{gathered}
$$

where,

$$
P x=\sum_{i, j=1}^{N} i \operatorname{Co}(i, j) \quad \text { and } \quad P y=\sum_{i, j=1}^{N} j \operatorname{Co}(i, j)
$$

and $\mathrm{Co}(\mathrm{i}, \mathrm{j})$ is the $(i, j)$ th element of the co-occurrence matrix. These parameters are also stored in database feature vector as Wavelet Co-occurrence Features (WCF).

\section{FUZZY LOGIC CLASSIFIER}

A Fuzzy system is a fuzzy logic based system. In this system fuzzy logic can be a basis for the representation of the various kinds of knowledge or it can model the interactions and relationships among the system variables. Fuzzy logic provides innovative tools to handle the complex and ill-defined systems where classical tools become unsuccessful. Fuzzy systems are universal approximators of non linear functions. Two aspects are important in fuzzy system one generating the best rule set and second tuning the membership functions. These should relate properly the independent and dependent variable.

Inputs to the fuzzy system are WSF and WCF features that are covered in this research. The outputs of the system are specific texture image. 
Fuzzy sets with Gaussian membership functions are used to define these input variables. These fuzzy sets can be defined using the following equation [16].

$$
\boldsymbol{\mu}(\boldsymbol{f})=e^{-0.5\left(\frac{f-m}{\sigma}\right)^{2}}
$$

where $\mathrm{m}$ is the mean of the fuzzy set and $\sigma$ is the standard deviation from the mean.

Rules for the fuzzy system are obtained by fuzzification of the numerical values from wavelet and co-occurrence matrix methods. The fuzzy sets corresponding to each texture feature are generated and maximum degree of membership will be $\mu=1$ for each fuzzy set. The standard deviation of feature values from the mean is calculated. Each texture feature is assigned to the fuzzy set with the maximum degree of membership.

Success rate is calculated using the results obtained after defuzzification.

\section{EXPERIMENTAL RESULTS AND DISCUSSIONS}

In this experiment twenty five texture images from the Brodatz texture [17] are used for classification [9]. These texture images are rotated through $0^{\circ}, 30^{\circ}, 60^{\circ}, 90^{\circ}, 120^{\circ}$, and $150^{\circ}$. Rotated textures are then sampled to $256 \times 256$, and $128 \times 128$ sizes. Out of 3300 random samples generated of each rotated texture image, 3000 are used for training the fuzzy classifier and remaining 300 are used to test the classifier. In this experiment pyramid structured type of DWT is used with $\mathrm{dB} 4$ as a wavelet filter.

Feature database is created using wavelet decomposed subbands up to fifth level of decomposition. Total number of subbands up to fifth level will be 20. Energy (1) and standard deviations (2) of each sub-band coefficients are calculated for each level and each of the rotated samples. These features are stored as WSF. Another feature database is obtained using eight co-occurrence features (3)-(10) by finding the co-occurrence matrix of original sample image and 4- sub-bands of the 1-level DWT coefficients co-occurrence matrix. These are stored as WCF. This way, maximum WSF will be $20 \times 2=40$ for five level decomposition and maximum WCF will be $5 \times 8=40$ for a sample.

The mean and standard deviation of WSF and WCF for 3300 samples each derived from rotated texture images are obtained. These are required for the fuzzy classification. Classification is carried out for following feature categories.

- WSF1- using Energy only of $1^{\text {st }}$ level, $2^{\text {nd }}$ level, $3^{\text {rd }}$ level, $4^{\text {th }}$ level, and $5^{\text {th }}$ level DWT decomposition using dB4.

- WSF2- using Standard Deviation only of $1^{\text {st }}$ level, $2^{\text {nd }}$ level, $3^{\text {rd }}$ level, $4^{\text {th }}$ level, and $5^{\text {th }}$ level DWT decomposition using dB4.

- WSF3- using Energy and Standard Deviation both of $1^{\text {st }}$ level, $2^{\text {nd }}$ level, $3^{\text {rd }}$ level, $4^{\text {th }}$ level, and $5^{\text {th }}$ level DWT decomposition using dB4.

- WCF1- using five co-occurrence features viz. contrast, energy, local homogeneity, cluster shade, and cluster prominence of whole image and four sub-bands of $1^{\text {st }}$ level DWT decomposition using $\mathrm{dB} 4$.
- WCF2- using eight co-occurrence features viz contrast, inverse difference moment, energy, norm entropy, local homogeneity, cluster shade, cluster prominence, \& maximum probability of whole image and four sub-bands of $1^{\text {st }}$ level DWT decomposition using dB4.

Performance of the above feature sets is tested with the help of a fuzzy classifier in terms of Success rate. Let $\mathrm{N}_{\mathrm{T}}$ be the no. of samples to be tested and out of that if the system correctly classifies $\mathrm{N}_{\mathrm{C}}$ times then success rate of the system for rule $i$ as a percentage is given as

$$
S_{R}=\frac{N_{C}}{N_{T}} x 100
$$

The best case as well as worst case analysis of each of the feature set is carried out for different angle rotated texture images to decide features performing well and giving excellent success rate. Average and minimum success rate for each category of feature set and number of features required for a particular sample size but for different angle texture images are studied and presented here.

Figure 1 shows that average success rate for every rotation angle with sample size of $256 \times 256$ is almost constant close to $100 \%$ irrespective of number of energy features (WSF1). Where as in case of $128 \times 128$ sample size for any angle it reaches to $100 \%$ from $3^{\text {rd }}$ to $4^{\text {th }}$ level of wavelet decomposition.

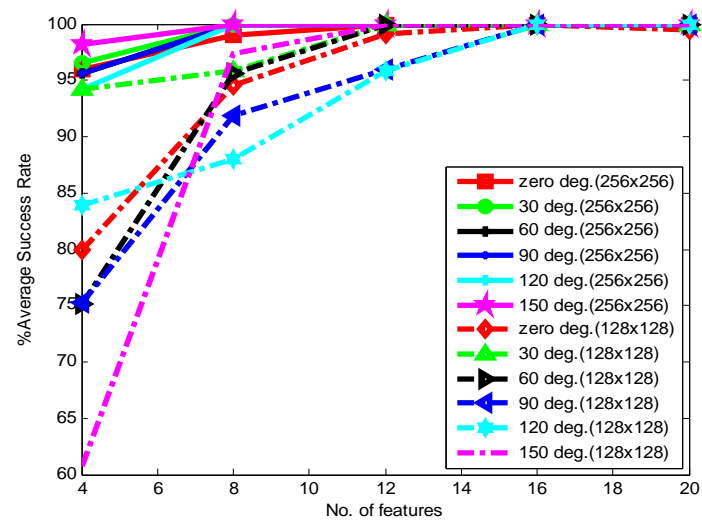

Figure 1: Average Success Rate with Energy only (WSF1)

In case of standard deviation (WSF2) features, average success rate becomes $100 \%$ from 8 number of features for $256 \times 256$ sample size and for all angles whereas for $128 \times 128$ samples it hardly reaches to $100 \%$ at $3^{\text {rd }}$ level as shown in figure 2 . 


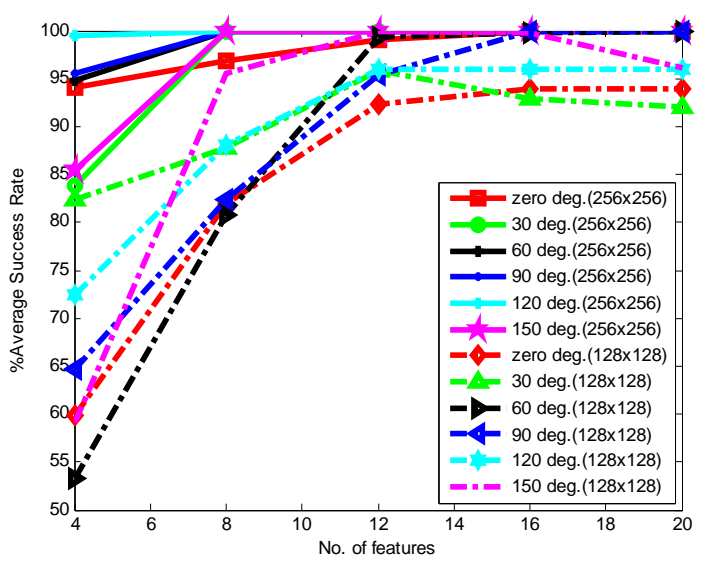

Figure 2: Average Success Rate with Standard Deviation only (WSF2)

When energy and standard deviation features are taken together (WSF3) then average success rate is above 93\% for $256 \times 256$ sample size at $1^{\text {st }}$ level while it becomes $100 \%$ for higher level of DWT decomposition for any angle of rotation. For $128 \times 128$ sample size average success rate becomes $100 \%$ from $3^{\text {rd }}$ level as shown in figure 3 .

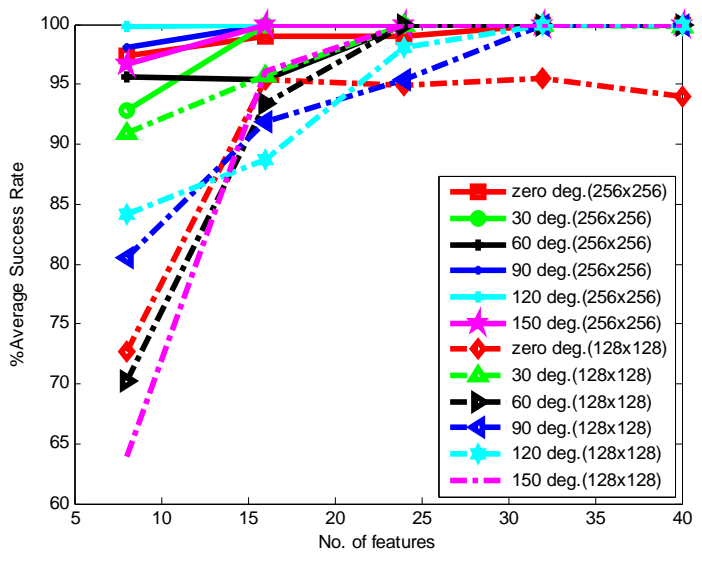

Figure 3: Average Success Rate with Energy + Standard Deviation (WSF3)

Figure 4 shows average success rate of the category of 5 cooccurrence features (WCF1) ranges above $89 \%$ at 5 numbers of features and reaches almost $100 \%$ from 10 numbers of features for $256 \times 256$ sample size and for any angle while it is maximum up to $94 \%$ at 25 features for $128 \times 128$ samples.

When whole set of co-occurrence features (WCF2) is taken then average success rate is $98 \%$ and above for $256 \times 256$ sample size. Whereas it is hardly $92 \%$ maximum for $128 \times 128$ sample size for any angle as depicted in figure 5 .

Worst case analysis is carried out to decide the minimum features required to get maximum success rate. It is presented in the table 1 shown below. From the table it is evident that energy features are most consistent and never fail to classify the texture images in any of the rotation angle. Also it is to be noted that for 256x256 sample size all feature set works properly but except WSF1 all other fails in or the other case for $128 \times 128$ sample size.

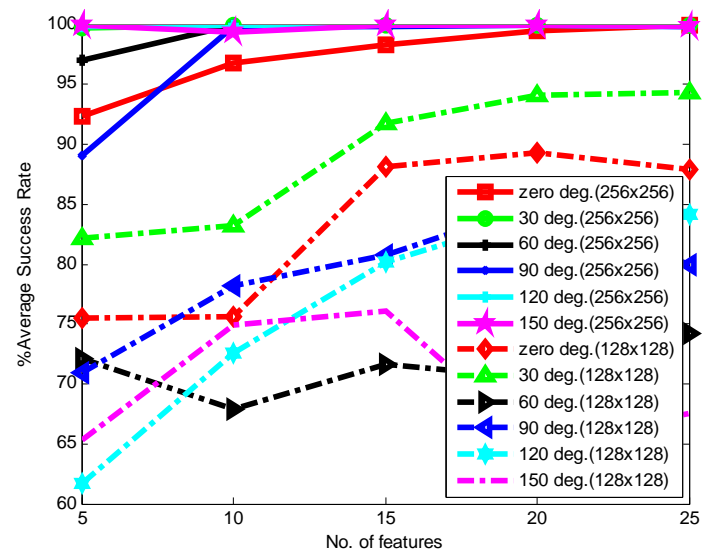

Figure 4: Average Success Rate of 5 Co-occurrence Features (WCF1)

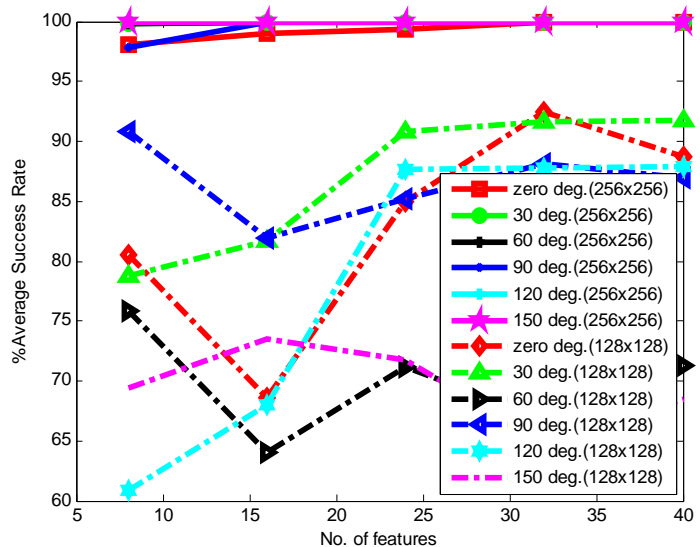

Figure 5: Average Success Rate with 8 Co-occurrence Features (WCF2)

\section{CONCLUSION}

In this study, Wavelet Statistical Features and Wavelet Cooccurrence Features are tested using fuzzy logic classifier for classification and retrieval of texture images under different angle of rotation. The performance is measured in terms of Success Rate. Best case and worst case analysis is carried out for the data. The best average as well as minimum number of features required is given by energy feature WSF1 for $256 \times 256$ sample size as well as $128 \times 128$ sample sizes. WCF features have not performed consistently in case of $128 \times 128$ samples. Worst case analysis showed that WSF features give excellent performance for $256 \times 256$ followed by $128 \times 128$ texture images.

The Best case as well as worst case analysis shows that the WCF2 offers excellent performance, for 256x256 sample size as compared to $128 \times 128$. These features are rotation invariant in the sense that for any angle of rotation it gives excellent performance. 
Table 1 Minimum Features Required

\begin{tabular}{|c|c|c|c|c|c|c|c|c|c|c|c|c|}
\hline \multirow[t]{2}{*}{ Features } & \multicolumn{6}{|c|}{ 256x256 sample size } & \multicolumn{6}{|c|}{ 128x128 Sample size } \\
\hline & $0^{0}$ & $30^{0}$ & $60^{0}$ & $90^{0}$ & $120^{0}$ & $150^{0}$ & $0^{0}$ & $30^{0}$ & $60^{0}$ & $90^{0}$ & $120^{0}$ & $150^{0}$ \\
\hline $\begin{array}{l}\text { Energy + } \\
\text { Standard } \\
\text { Deviation(WSF3) }\end{array}$ & 6 & 10 & 10 & 8 & 6 & 10 & $\mathrm{xx}$ & 20 & 18 & 27 & 26 & 18 \\
\hline Energy(WSF1) & 3 & 4 & 6 & 6 & 6 & 6 & 7 & 11 & 9 & 15 & 15 & 8 \\
\hline $\begin{array}{l}\text { Standard } \\
\text { Deviation(WSF2) }\end{array}$ & 3 & 6 & 7 & 6 & 4 & 7 & $\mathrm{xx}$ & 11 & 12 & 13 & $\mathrm{xx}$ & 10 \\
\hline $\begin{array}{l}5 \text { Co-occurrence } \\
\text { (WCF1) }\end{array}$ & 8 & 5 & 8 & 9 & 5 & 5 & $\mathrm{XX}$ & $\mathrm{XX}$ & $\mathrm{XX}$ & $\mathrm{XX}$ & $\mathrm{xX}$ & $\mathrm{xX}$ \\
\hline $\begin{array}{l}8 \text { Co-occurrence } \\
\text { (WCF2) }\end{array}$ & 7 & 6 & 8 & 10 & 6 & 6 & $\mathrm{XX}$ & XX & $\mathrm{XX}$ & $\mathrm{XX}$ & $\mathrm{XX}$ & $\mathrm{XX}$ \\
\hline
\end{tabular}

$\mathrm{xx}$ means features under consideration are not sufficient to classify all the textures

In the current study, features tested for only twenty five textures for rotation invariance which give excellent results with 256x256 sample size.

\section{REFERENCES}

[1] R. M. Haralick, K. Shanmugam, and I. Dinstein, 1973 Textural features for image classification, IEEE Trans. on Systems, Man, and Cybernetics, SMC-3, 610-621.

[2] J. Weszka, C. Dyer, and A. Rosenfeld, 1976 A comparative study of texture measures for terrain classification, IEEE Trans. on Sys., Man. and Cyb., SMC-6(4).

[3] Y. Wan, J. Du, D. Huang, Z. Chi, Y. Cheung, X. Wang, G. Zhang, 2004, Bark Texture Feature Extraction Based on Statistical Texture Analysis, In Proceedings of 2004 Int. Sympo. On Intelligent multimedia, Video \& Speech processing, Hong Kong.

[4] B. S. Manjunath, and W. Y. Ma, 1996 Texture features for browsing and retrieval of image data, IEEE Trans. on PAMI, Vol. 18, No. 8, 837-842.

[5] I. Daubechies, 1990 The wavelet transform, time-frequency localization and signal analysis, IEEE Trans. on Information Theory, Vol. 36, 961-1005.

[6] S. G. Mallat, 1989 A theory for multi-resolution signal decomposition: the wavelet representation, IEEE Trans. on PAMI, Vol. 11, 674-693.

[7] J. R. Smith and S. F. Chang, 1994, Transform features for texture classification and discrimination in large image databases, In Proceedings of IEEE International Conference on Image Processing.

[8] R.O. Duda, P.E. Hart, D.G. Stork, 2006, Pattern Classification, John Wiley and Sons, Second Edition.

[9] E.Avci, 2007 An expert system based on Wavelet Neural Network-Adaptive Norm Entropy for scale invariant texture classification, Journal on Experts Systems with Applications, Vol.32, 919-926, Elsevier.

[10] I. Turkoglu and E.Avci, 2008 Comparison of wavelet-SVM and Wavelet-adaptive network based fuzzy inference system for texture classification, Journal on Digital Signal Processing, Vol.18, 15-24, Elsevier.

[11] G. Schaefer, M. Zavisek, T.Nakashima, 2009 Thermography based breast cancer analysis using statistical features and fuzzy classification, Journal of Pattern Recognition, Vol. 47, 1133-1137, Elsevier.

[12] S.M. Mukane, S.R. Gengaje, and D.S. Bormane, 2011 On Scale Invariance Texture Image Retrieval using Fuzzy Logic and Wavelet Co-occurrence based Features, International Journal of Computer Applications, Vol.18, No.3, 10-17.

[13] S.M. Mukane, D.S. Bormane, and S.R. Gengaje, 2011 On Size Invariance Texture Image Retrieval using Fuzzy Logic and Wavelet based Features, International Journal of Applied Engineering Research, Vol. 6, No. 6, 1297-1310.

[14] M. Kokare, P. K. Biswas, and B. N. Chatterji, 2006 Rotation-invariant texture image retrieval using rotated complex wavelet filters, IEEE Trans. on Systems, Man, and Cybernetics-Part B: Cybernetics, Vol.36, No.6, 1273-1282.

[15] S. Arivazhagan, L. Ganesan, T. Subash Kumar, 2006 Texture classification using ridge let transform, Pattern Recognition Letters, Vol.27, 1875-1883, Elsevier.

[16] P.M. Pawar and R. Ganguli, 2003 Genetic fuzzy system for damage detection in beams and helicopter rotor blades, Computer methods in applied mechanics and engineering, Vol.192, 2031-2057, Elsevier.

[17] P. Brodatz, 1996, Textures: A Photographic Album for Artists and Designers, New York: Dover. 